\title{
POSITIONS OF EXTRAGALACTIC RADIO SOURCES \\ FROM VERY LONG BASELINE INTERFEROMETRY
}

\author{
(Invited Paper) \\ C. C. COUNSELMAN III \\ Massachusetts Institute of Technology, Cambridge, Mass. 02139, U.S.A.
}

\begin{abstract}
The positions of extragalactic radio sources have been determined by very-long-baseline interferometry with uncertainties smaller than 0 ". 1 , and fundamental limits on the accuracy of VLBI astrometry have not yet been reached. Technical improvements within the next five years may enable positions to be determined by VLBI with uncertainties of 0 " 005 .
\end{abstract}

\section{Introduction}

Extragalactic objects can provide an excellent approximation to an inertial reference frame. Thus it is important to determine their positions as accurately as possible. By very-long-baseline interferometry (VLBI), the positions of extragalactic radio sources can now be determined with uncertainties smaller than 0 ." 1 , as demonstrated by the consistency between results obtained by different groups of observers who used different very-long-baseline interferometers and significantly different techniques.

\section{VLBI Techniques}

VLBI requires no real-time communication or connection between the separate antennas which form the interferometer.* The signal received at each antenna is converted to a low frequency and recorded on tape with a time base provided by the local station clock. Tapes which have been recorded at separate antennas simultaneously observing the same radio source are later brought together and played back to determine the difference between the time bases, or readings of the independent station clocks, for which the crosscorrelation between the recorded signals is greatest. Except for small instrumental and atmospheric delays, aberration, and random noise, the resulting value of this time-difference is given by

$$
\Delta \tau=\frac{1}{c} \mathbf{B} \cdot \hat{\mathbf{s}}+\Delta \tau_{\text {clock }}
$$

where $c$ is the speed of light; $\mathbf{B}$ is the vector position of one antenna with respect to the other, known as the interferometer 'baseline' vector; $\hat{\mathbf{s}}$ is a unit vector in the direction of the source; and $\Delta \tau_{\text {clock }}$ stands for the error of synchronization between the two station clocks.

* This feature, rather than the physical distance between the antennas, distinguishes VLBI from conventional, connected-element interferometry. 
If the baseline vector $\mathbf{B}$ and the clock offset $\Delta \tau_{\text {clock }}$ could be determined independently, then an unknown source-direction unit-vector $\hat{\mathbf{s}}$ could be determined by making only two observations several hours apart, using the Earth's rotation to obtain different projections of $\mathbf{B}$ upon $\hat{\mathbf{s}}$. (Note that the baseline vector should not be nearly parallel to the Earth's spin axis. Neither should it lie too close to the equatorial plane; if it did, the accuracy of determinations of the declinations of near-equatorial sources would be degraded.) It is possible to determine simultaneously all 3 components of an unknown baseline vector, the clock offset $\Delta \tau_{\text {clock }}$ as a continuous function of time, and both coordinates of all sources observed by a 2-station interferometer if at least 3 well-distributed sources are observed for several hours. In this determination an origin of longitude or right ascension must be defined by some external means. Usually the right ascension of one of the sources, or the mean of several, is fixed. However, declinations are determined absolutely with respect to the equator of date.

The discussion up to this point has been appropriate for VLBI observations using wide effective bandwidth, for which accurate and unambiguous determination can be made of the group-delay difference between the signals received at the two antennas. VLBI observations can also be done usefully with narrow bandwidth, such that only the time-variation of the phase-delay difference (sometimes called the 'fringe phase'; the time-derivative of fringe phase is often called the 'fringe rate') can be determined with significant accuracy. With only phase-delay or rate observations it is impossible to determine $\Delta \tau_{\text {clock }}$ and the component of B parallel to the Earth's spin axis, and it is impossible to determine the declinations of all of the sources simultaneously with the length of the equatorial projection of $\mathbf{B}$. The sensitivity of these observables to the declination $\delta$ of a source varies as $\sin \delta$, approaching zero at the equator. Usually a priori information is used to constrain the declination of a near-equatorial source, so that the declinations of the remaining sources and the equatorial projection of $\mathbf{B}$ can be determined from narrow-band VLBI data.

A general introduction to VLBI by Cohen (1973), a description of a VLBI taperecorder system by Clark (1973), a mathematical analysis of the tape-processing by Moran (1973), and other related papers are collected in the September 1973 Special Issue on Radio and Radar Astronomy of the Proceedings of the IEEE.

\section{Accuracy: Present and Future}

To assess the present accuracy of source-position determinations by VLBI, I have compared the results obtained independently by two different groups of observers who used different very-long-baseline interferometers and significantly different techniques which are summarized in Table I. One set of results was obtained by T. A. Clark of the Goddard Space Flight Center of the U.S. National Aeronautics and Space Administration, A. E. E. Rogers and H. F. Hinteregger of Haystack Observatory, and C. C. Counselman III, C. A. Knight, D. S. Robertson, I. I. Shapiro, and A. R. Whitney of the Massachusetts Institute of Technology; they were first reported 
TABLE I

Parameters of two independent determinations of radio source positions by VLBI. See text for explanations

\begin{tabular}{lll}
\hline $\begin{array}{l}\text { Participating } \\
\text { Organizations }\end{array}$ & $\begin{array}{l}\text { GSFC, } \\
\text { Haystack, } \\
\text { MIT }\end{array}$ & $\begin{array}{l}\text { JPL, } \\
\text { INTA }\end{array}$ \\
Stations used & $\begin{array}{l}\text { Haystack, } \\
\text { Goldstone, } \\
\text { NRAO, } \\
\text { OVRO }^{\mathrm{a}}\end{array}$ & $\begin{array}{l}\text { Goldstone, } \\
\text { Madrid }\end{array}$ \\
& $3900^{\mathrm{b}}$ & \\
& 4 & 8400 \\
$\begin{array}{l}\text { Baseline length }(\mathrm{km}) \\
\text { Wavelength }(\mathrm{cm}) \\
\text { Observable }\end{array}$ & Group delay & 13 \\
\hline
\end{tabular}

a The National Radio Astronomy Observatory in Green Bank, West Virginia and the Owens Valley Radio Observatory in California were used only in 1969.

${ }^{b}$ Haystack-Goldstone.

by Clark et al. (1973), and are presented in detail by Rogers et al. (1973). These results were based on four separate periods of observation in 1969 and 1972, using mainly the 37-m diameter antenna of the Haystack Observatory in Tyngsboro, Massachusetts and the 64-m diameter antenna of the NASA Deep Space Net station in Goldstone, California. The observations were of group delay, for bandwidths of $35 \mathrm{MHz}$ in 1969 and $23 \mathrm{MHz}$ in 1972 , centered on a frequency near $8 \mathrm{GHz}(\lambda \approx 4 \mathrm{~cm})$.

These source-position results are compared to the preliminary positions reported at this Symposium by J. L. Fanselow, P. F. MacDoran, J. D. Thomas, D. J. Spitzmesser, L. Skjerve, and J. G. Williams of the Jet Propulsion Laboratory, and J. Urech of the Instituto Nacional de Technica Aeroespacial, Madrid, Spain. There are 8 sources whose positions have been determined by both groups : 3C 84, 3C120, OJ287, 4C 39.25, $3 C 345$, PKS2134+00, VRO42.22.01, and 3C454.3. For these 8 sources the rms difference between the right ascension determinations of the two groups was 0.005 , or 0.08 . Excluding the low-declination sources 3C120 and PKS2134+00 from comparison (because the fringe-rate observable used by the JPL-INTA group is insensitive to declination at the equator), the rms difference between the declination determinations of the two groups is 0 ".07. It is perhaps worth noting that the rms disagreement between the two groups' results is actually smaller than the published uncertainties; since the two groups worked completely independently this may signify that their quoted uncertainties are pessimistic.

What determines these uncertainties, and what improvements can we expect within a few years? The main sources of error are (i) the atmosphere, (ii) instrumental drifts, and (iii) geophysical-astronomical model inadequacies. I will discuss these in turn:

(i) Atmosphere. The equivalent electrical path length of the earth's neutral atmosphere, at $30^{\circ}$ elevation, is about $5 \mathrm{~m}$, but the a priori uncertainty is probably less 
than $60 \mathrm{~cm}$. Assuming that the variations of atmospheric path length at opposite ends of the interferometer baseline are uncorrelated (any positive correlation only reduces the effect on the interferometric delay observable), we find that the equivalent source position uncertainty for a $4000-\mathrm{km}$ baseline is about 0 ".04. This uncertainty may be reduced significantly by modeling and making use of observations taken over a wide range of elevations to determine parameters in a model of the atmosphere simultaneously with the source coordinates, baseline vector components, and clocksynchronization parameters. However, a new method (Schaper et al., 1970) involving microwave radiometric measurements of the emission of atmospheric water vapor (the constituent responsible for most of the path-length uncertainty) appears capable of reducing the uncertainty in atmospheric path-length at $30^{\circ}$ to less than $3 \mathrm{~cm}$, which corresponds to an uncertainty in source position of only 0.002 .

Depending on the radio frequency used in VLBI observations, the ionosphere may constitute a significant error source. At the $8-\mathrm{GHz}$ frequency of the HaystackGoldstone observations, the uncertainty introduced by the ionosphere is on the order of 0.005 , but this uncertainty could easily be reduced to a negligible level by using a higher frequency (e.g., several antennas exist which are suitable for VLBI observations at $15 \mathrm{GHz}$ ) or by making simultaneous observations at a lower frequency and using the $f^{-2}$ dependence of ionospheric delay to calibrate this delay.

(ii) Instrumental drifts. Until recently, relatively little attention has been paid to measuring and correcting for variations in instrumental delays due to varying temperature, etc., in VLBI equipment. (Delay calibration of short-baseline instrumentation has received much more attention, because the effects of instrumental delay on source-position results increase with decreasing baseline length.) However, delaycalibration equipment suitable for VLBI has now been developed (by A. E. E. Rogers at Haystack Observatory) and installed at several antennas. With this equipment, drifts on the order of $1 \mathrm{~ns}$ in delay, equivalent to 0 ".015 in position for a $4000-\mathrm{km}$ baseline, have been measured, and should now be removable within less than 100 ps or 0."0015.

(iii) Geophysical-astronomical model. In this category I include the problem of calculating the relative motions of the antennas due to solid-Earth tides, and the rotation of the earth. The effects of tides are clearly evident in Haystack-Goldstone VLBI results: a significant reduction in the post-fit residuals was obtained when a simple tidal model was included in the data-reduction program for the first time. Crustal motions on a time scale much longer than a day do not interfere with the determination of souce positions because the baseline can always be re-determined separately on each day of observation. It is not yet clear what problems remain to be solved in the area of Earth rotation, but the ability of group-delay VLBI to determine both the source positions and the vector baseline on a time scale of hours will be important as these problems are sorted out in the future. If a set of extragalactic sources is used arbitrarily to define a reference frame, then the rotation of the baseline vector with respect to this frame may be determined, simultaneously with clocksynchronization error, as quickly as the antennas can be moved through a well- 
distributed set of 4 sources. It seems likely that VLBI will play an important part in future determinations and improvements of theoretical models of precession, nutation, polar motion, and the variation of UT1.

\section{Recent Results}

Since the results compared in the preceding section were based on observations made in some cases as much as 4 years ago, one might ask what has been achieved in more recent, presumably more accurate, work. Preliminary results have been obtained from a series of VLBI observations made by the GSFC-Haystack-MIT VLBI group in October, 1972, using 2 antennas at Haystack Observatory and 2 antennas at NRAO, Green Bank, in a differential-interferometric mode. In this mode, a pair of sources is observed simultaneously using the same station clock for both sources at each end of the long baseline. The relative positions of the sources may thus be determined free from effects of clock instability. Using this data, Knight (1973) has made an independent determination for each of 8 days of observation, spanning 4 weeks and under very different weather conditions, of the position of quasar $3 \mathrm{C} 279$ relative to another quasar, 3C 273.* The rms scatter of these 8 independent determinations of differential right ascension was only 0.02. Declination scatter was much greater, partly because only fringe phase observations were used and $3 \mathrm{C} 279$ is only $5^{\circ}$ from the equator: the rms was 0 ".08. Significant systematic errors which would not be revealed by the day-to-day scatter of position determinations may, of course, be present. Comparisons of these with other recent results will be necessary before the true uncertainties can be estimated.

\section{Conclusion}

It seems reasonable to expect that within five years the uncertainties of radio source position determinations by VLBI will be as small as 0 ."005. However, this low level of uncertainty will probably apply only to the relative positions of these sources, i.e., to their coordinates in a system defined by these sources alone, without reference to the ecliptic or the dynamical equinox. The important tasks of determining the orbits of the Earth, Moon, and planets, and the rotation of the Earth with respect to this extragalactic reference system to comparable levels of uncertainty will require more time. For these tasks, VLBI will also be important. Differential interferometric observations of extragalactic radio sources and spacecraft on the surfaces of, or in orbit around, the Moon and planets may be used to determine Earth-Moon and Earthplanet directions relative to the extragalactic sources (Counselman et al., 1972; Counselman et al., 1973).

\footnotetext{
* This pair was also observed on several other days, when the Sun was near $3 \mathrm{C} 279$, in order to measure the solar gravitational deflection.
} 


\section{References}

Clark, B. G. : 1973, Proc. IEEE 61, 1242-1248.

Clark, T. A., Counselman, C. C., III, Hinteregger, H. F., Knight, C. A., Robertson, D. S., Rogers, A. E. E., Shapiro, I. I., and Whitney, A. R.: 1973, Bull. Am. Astron. Soc. 5, 30.

Cohen, M. H.: 1973, Proc. IEEE 61, 1192-1197.

Counselman, C. C., III, Hinteregger, H. F., and Shapiro, I. I.: 1972, Science 178, 607-608.

Counselman, C. C., III, Hinteregger, H. F., King, R. W., and Shapiro, I. I. : 1973, Science 181, $772-774$.

Knight, C. A.: 1973, personal communication.

Moran, J. M.: 1973, Proc. IEEE 61, 1236-1242.

Rogers, A. E. E., Counselman, C. C., III, Hinteregger, H. F., Knight, C. A., Robertson, D. S., Shapiro, I. I., and Whitney, A. R.: 1973, Astrophys. J. 186, in press.

Schaper, L. W., Jr., Staelin, D. H., and Waters, J. W.: 1970, Proc. IEEE 58, 272-273.

\section{DISCUSSION}

Elsmore: Am I right in thinking that atmospheric effects provide the limit to the positional accuracies that VLBI techniques can achieve?

Counselman: I think so, assuming that instrumental phase calibrators, which have been installed only recently, prove successful in reducing instrumental path-length uncertainty to less than a centimeter. If water-vapor radiometry techniques enable us, as we hope and expect, to determine atmospheric path length differences within $2 \mathrm{~cm}$, then we should be able to determine source positions within milliseconds of arc using a $4000-\mathrm{km}$ interferometer baseline. Of course, at such a level of angular resolution, source structure becomes important and must be specified in order to specify a source position.

Mulholland: Your determination of the variation of UT1 consistent with USNO to 5 ms should be very satisfying, since that is near the uncertainty level in the PZT determinations.

Counselman: Yes, the agreement between our determinations and those of the USNO is close enough to be satisfying, but not so close that you would be suspicious. Identical comments apply to our determinations of polar motion in relation to those by the BIH. 